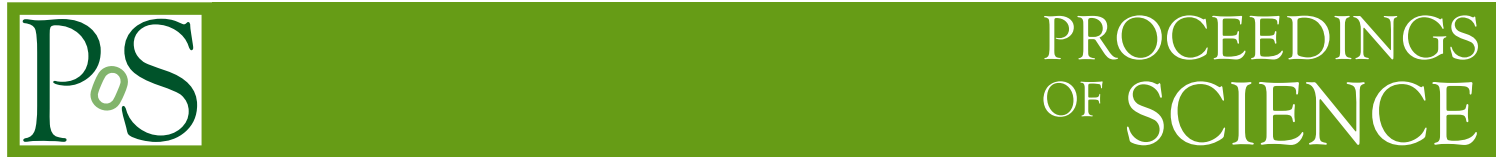

\title{
Jet Quenching with CMS
}

\section{Pelin Kurt* University of Illinois at Chicago}

E-mail: pkurtecern.ch

\begin{abstract}
An overview of the most recent results on jet quenching physics obtained using $\mathrm{PbPb}$ collision data collected with the CMS experiment at $\sqrt{s_{\mathrm{NN}}}=2.76 \mathrm{TeV}$ will be presented. These measurements make use of many different observables, including momentum imbalance of dijet and photon-jet events, nuclear modification factor $R_{A A}$, as well as jet fragmentation functions, jet shapes, and flavor dependence of jet quenching. All these measurements in $\mathrm{PbPb}$ collisions will be presented and compared measurements from $2.76 \mathrm{TeV}$ pp collisions to study effects of parton energy loss in the hot and dense medium. Since many of these observables have low correlation to one-another they serve as useful independent consraints to the nature of the parton energy loss mechanism.
\end{abstract}

The European Physical Society Conference on High Energy Physics -EPS-HEP2013 18-24 July 2013

Stockholm, Sweden

\footnotetext{
*Speaker.
} 


\section{Introduction}

Heavy ion collisions at the Large Hadron Collider (LHC) present a great opportunity to study the phases of nuclear matter predicted by Quantum Chromodynamics (QCD), the theory of the strong interaction. Jets originating from hard scatterings of partons are a powerful probe of the hot, dense matter created in heavy-ion collisions. This medium is commonly referred to as a QuarkGluon Plasma (QGP). The partons are expected to lose energy while traversing the medium via elastic processes (collisional parton energy loss) or inelastic processes (radiative parton energy loss) [1]. At RHIC, indirect measurements of energy loss in the medium ("jet quenching") have been made by studying high momentum jet fragmentation products. More recently, the Compact Muon Solenoid (CMS) [2] detector has been used to study for parton energy loss in the QGP with leading particle and jet coincidence measurements. We present some selected measurements related to parton energy loss in $\mathrm{PbPb}$ collisions at a nucleon-nucleon center-of-mass energy of $\sqrt{S_{\mathrm{NN}}}=2.76 \mathrm{TeV}$ collected in 2010 and 2011 using the CMS detector.

\section{Experimental Techniques}

In order to study medium-induced modifications, we first perform a measurement in $\mathrm{PbPb}$ collisions where the medium is present, and then compare them to the same measurement in pp collisions. The reference measurement in pp collisions is performed at the same centre-of-mass energy using the data sample from 2013, which yields a kinematic reach for the jets similar to that in the $\mathrm{PbPb}$ data. The capabilities of the CMS detector allows us to investigate various hard probes, using excellent tracking, calorimeter, and muon systems which cover a large range in pseudorapidity. All of these detectors have sufficient granularity and resolution to function well even in the highest multiplicities encountered in $\mathrm{PbPb}$ collisions. It is also important to note that heavy ions are extended objects, so the impact parameter is an important characterization of the events. The centrality of the collisions is defined as a fraction of the total nucleus-nucleus inelastic cross section, with $0 \%$ denoting the most central collisions with impact parameter 0 , and $100 \%$ - the most peripheral collisions. In these analyses, centrality was determined from minimum bias events based on the total energy from both forward hadronic calorimeters [3].

\section{Results}

In comparison to pp collisions, a large fraction of dijets with imbalanced transverse momentum is observed in $\mathrm{PbPb}$ collisions at $\sqrt{s_{\mathrm{NN}}}=2.76 \mathrm{TeV}$. To characterize the dijet momentum balance (or imbalance) quantitatively, we use the asymmetry ratio $A_{j}=\frac{p_{T_{1}}-p_{T_{2}}}{p_{T_{1}}+p_{T_{2}}}$, where $p_{T_{1}}$ is the transverse momentum of the leading jet and required to be $p_{T_{1}}>120 \mathrm{GeV}$, and $p_{T_{2}}$ is the transverse momentum of the subleading jet in the opposite hemisphere with $p_{T_{2}}>30 \mathrm{GeV}$. The centrality dependence of $A_{j}$ for $\mathrm{PbPb}$ collisions is shown in Fig. 1, in comparison to PYTHIA+HYDJET simulations. The most peripheral events are also compared to results from pp collisions at $\sqrt{s_{\mathrm{NN}}}=2.76 \mathrm{TeV}$, where the same jet algorithm is used. The shape of the dijet momentum balance distribution gradually changes with collision centrality, towards a larger imbalance. In contrast, dijets from simulated 


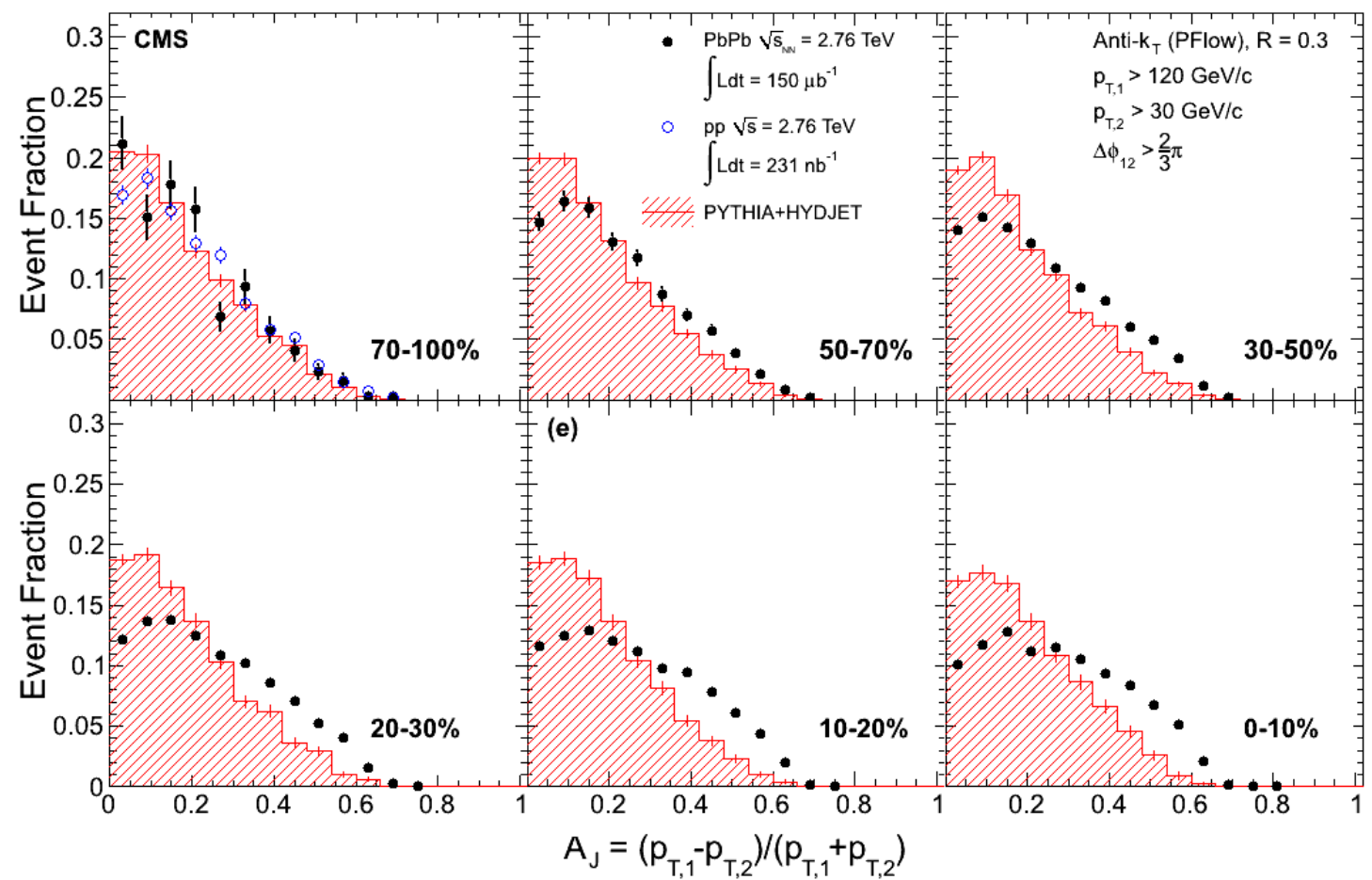

Figure 1: Dijet asymmetry ratio, $A_{J}$, for leading jets of $p_{T_{1}}>120 \mathrm{GeV} / \mathrm{c}$ and subleading jets of $p_{T_{2}}>$ $30 \mathrm{GeV} / \mathrm{c}$ with a selection of $\Delta \phi>2 \pi / 3$ between the two jets [4]. Results from data are shown as points, while the histogram shows the results for PYTHIA dijets embedded into HYDJET PbPb simulated events. Data from pp collisions at $2.76 \mathrm{TeV}$ are shown as open points in comparison to $\mathrm{PbPb}$ results of $70-100 \%$ centrality. The error bars represent the statistical uncertainties.

PYTHIA events only exhibit a modest broadening, even when embedded in the highest multiplicity $\mathrm{PbPb}$ events.

In a more detailed study of the parton energy loss mechanism [3], CMS has investigated the redistribution of the quenched jet energy using the transverse momentum balance of charged tracks projected onto the direction of the leading jet axis, defined as $p_{T}^{\|}=\sum_{\mathrm{i}}-p_{T}^{\mathrm{i}} \cos \left(\phi_{\mathrm{i}}-\phi_{\text {Leading Jet }}\right)$, where the sum is evaluated over all tracks with $p_{T}>0.5 \mathrm{GeV} / \mathrm{c}$ and $|\eta|<2.4$. The results were then averaged over the event ensemble to obtain $\left\langle p_{T}^{\|}\right\rangle$. Fig. 2 (left) shows $\left\langle p_{T}^{\|}\right\rangle$as a function of $A_{J}$ for the most central $\mathrm{PbPb}$ collisions (0-30\%). Even for events with a very unbalanced dijet (large $A_{J}$ values), the total summed projected momentum of all the included tracks (solid circles) is close to zero. The colored bands (with vertical bars for statistical uncertainties) show the summed momentum for tracks restricted to specific $p_{T}$ ranges. The main conclusion here is that a large fraction of the momentum balance of the jets in unbalanced events is carried by low- $p_{T}$ particles at large radial distance to the jet axis [3].

While studies using dijets benefit from the large dijet production cross section, the energy loss of both partons makes the determination of the amount of energy lost by each parton more difficult. Correlations between isolated photons and jets have been proposed in the literature as the "golden channel" to study jet energy loss. This is because the photon retains the kinematic information of 


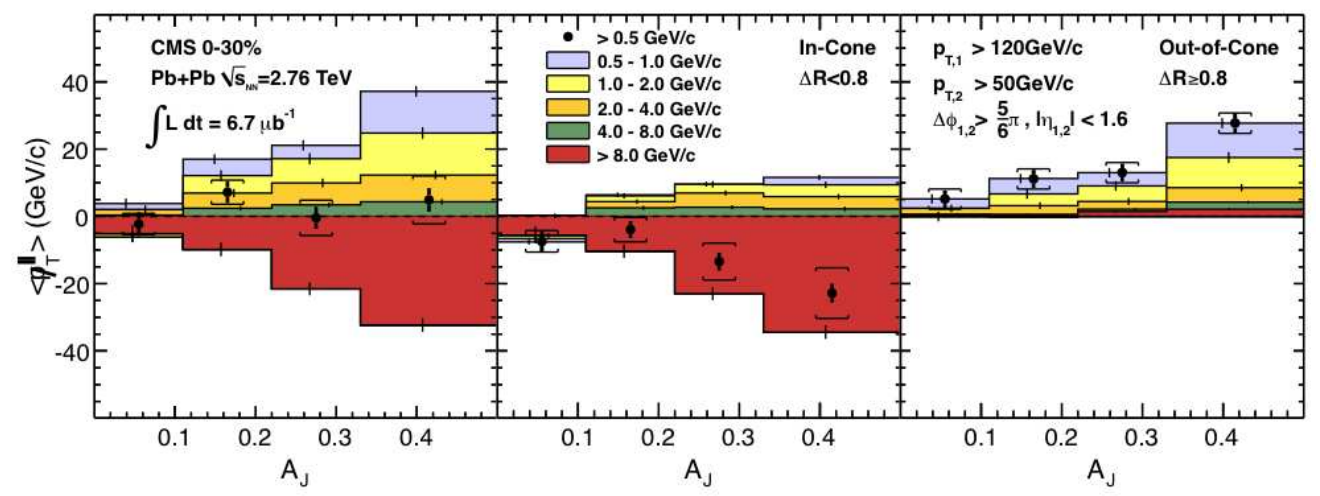

Figure 2: (Left) Average missing transverse momentum, $\left\langle p_{T}^{\|}\right\rangle$, for tracks with $p_{T}>0.5 \mathrm{GeV} / \mathrm{c}$, projected onto the leading jet axis (solid circles). The $\left\langle p_{T}^{\|}\right\rangle$values are shown as a function of dijet asymmetry for the $30 \%$ most central events. (Middle) The $\left\langle p_{T}^{\|}\right\rangle$values as a function of $A_{J}$ inside $(\Delta R<0.8)$ one of the leading or subleading jet cones. (Right) $\left\langle p_{T}^{\|}\right\rangle$outside $(\Delta R>0.8)$ the leading and subleading jet cones. For the solid circles, vertical bars and brackets represent the statistical and systematic uncertainties, respectively. Colored bands, with vertical bars for statistical uncertainties, show the contribution to $\left\langle p_{T}^{\|}\right\rangle$for five ranges of track $p_{T}[3]$.

the hard scattering since it is not expected to interact with the medium. In addition, the energy resolution for photons is better, making the photon an ideal object against which to compare the jet. In order to quantify any angular broadening, the $\mathrm{PbPb}$ data were compared to both pp data and a PYTHIA+HYDJET reference which included the effect of the underlying $\mathrm{PbPb}$ event but no parton energy loss. Similar to dijet events, no angular broadening was observed beyond that seen in the pp data and MC reference at all centralities. Further details about the measurement of isolated-photon+jet correlations in $\sqrt{s_{\mathrm{NN}}}=2.76 \mathrm{TeV}$ pp and $\mathrm{PbPb}$ collisions with CMS can be found elsewhere [5].

Another interesting analysis is a measurement of the jet flavor dependence of the jet quenching, which is expected to depend on the flavor of the initial parton. Gluon jets are expected to be quenched more strongly than light quark jets due to the larger color factor for gluon emission from gluons than from quarks. On the other hand, jets initiated by heavy quarks, particularly bottom quarks, are expected to radiate less than light ones. To measure this flavour dependence, the CMS collaboration has applied a b-jet identification algorithm for the first time in heavy ion collisions to perform such a measurement [6]. The purity of b-jet tagging is determined from template fits to the secondary vertex invariant mass distribution, and the efficiency of the secondary vertex tagging is estimated in a data- driven technique. The fraction of b-jet among inclusive jets is measured as a function of transverse momentum after purity and efficiency corrections in the range of 80 $<p_{T}^{\text {jet }}<200 \mathrm{GeV} / \mathrm{c}$. The fraction of $\mathrm{b}$-jets in $\mathrm{pp}$ and $\mathrm{PbPb}$ collisions are comparable, with no $p_{T}$ dependence, indicating that $\mathrm{b}$-quark jets are quenched similar to the light quark jets, i.e. the $R_{A A}$ value is $\approx 0.5 \pm 0.21$ (syst) [7]. These measurements have significant statistical uncertainties at present. The use of a larger pp data sample, along with a more precise calibration of tagging efficiencies and fit template shapes, may lead to significant improvements in precision in the future. 
A differential measurement to test the effects of the medium using a jet shape observable is performed using the CMS experiment. The jet shapes are a sensitive tool for the characterization of the parton-medium interactions by utilizing the energy flow inside the jet. Predictions have been made that the jet shapes will become wider due to quenching effects [8]. We present the first experimental test of this prediction. The jet shapes can be studied by using an integrated or a differential distribution. The differential jet shapes are defined as the average fraction of the transverse momentum contained inside an annulus of an inner radius $r_{\mathrm{a}}=r-\delta r / 2$ and an outer radius $r_{\mathrm{b}}=r+\delta r / 2$ as specified in the following equation

$$
\rho(r)=\frac{1}{\delta r} \frac{\sum_{r_{\mathrm{a}}<r_{i}<r_{\mathrm{b}}} p_{\mathrm{T}, i}}{\sum_{r_{i}<R} p_{\mathrm{T}, i}},
$$

where $\delta r$ is used as the annulus size, which is 0.05 . The sums run over the reconstructed particles, with the distance $r_{i}=\sqrt{\left(\eta_{i}-\eta_{j e t}\right)^{2}+\left(\phi_{i}-\phi_{j e t}\right)^{2}}$ relative to the jet axis described by $\eta_{j e t}, \phi_{j e t}$. A small cone size $(\mathrm{R}=0.3)$ was used for the jet reconstruction in order to suppress the underlyingevent contribution in the high multiplicity $\mathrm{PbPb}$ environment. All charged particles that pass a $p_{T}>1 \mathrm{GeV} / \mathrm{c}$ threshold are used to reconstruct jet shapes. Corrections for the tracking inefficiency are applied. In order to subtract the heavy-ion background, an $\eta$-reflection technique [9] was used. In order to understand the medium-parton interactions we compare the $\mathrm{PbPb}$ jet shapes results with those obtained from a pp reference. The measured differential jet shapes for $\mathrm{PbPb}$ and pp reference data are presented in Fig. 3 for different centrality bins, ranging from most-peripheral (70-100\%) to most central (0-10\%). The bottom panel shows the ratio of the $\mathrm{PbPb}$ jet shapes to the jet shapes for a pp reference obtained for the respective selections. Deviations from unity indicate modification of jet structure in the nuclear medium. We note that the jet shape spectra are normalized to unity. As a result, an excess at one distance $r$ from the jet axis has to be compensated by a depletion in another region. In all centrality classes, the ratios have a concave shape, which is more pronounced in the more central collisions. In central collisions (10-30\% and 0-10\%), an excess at large radius $r>0.2$ emerges, indicating a moderate broadening of the jets in the medium. This result is consistent with previous studies in CMS which find that the energy that the jets lose in the medium is redistributed at large distances from the jet axis outside the jet cone [3].

The observed enhancement in jet shapes results is consistent with the excess in the low $p_{T}$ part of jet fragmentation functions, formulated with $\xi$ and defined as $\xi=\ln \frac{1}{z} ; \mathrm{z}=\frac{p_{\|}^{\text {track }}}{p_{T}^{\text {jet }}}$ where $p_{\|}^{\text {track }}$ is the momentum component of the track along the jet axis, and $p_{T}^{j e t}$ is the transverse momentum of the reconstructed jet, respectively [10]. The jet shape and fragmentation function results indicate that suppression of intermediate $p_{T}$ particles is transported to larger radii with low $p_{T}$ particles enhancements by energy redistribution.

The CMS collaboration has performed many interesting measurements in $\mathrm{PbPb}$ collisions. All these measurements in $\mathrm{PbPb}$ collisions are presented and compared with observations in 2.76 $\mathrm{TeV}$ pp collisions to probe for distortions from energy loss in the hot and dense medium. Since many of these observables have low correlation to one-another they serve as useful independent confirmations of the quenching properties, and indicate a consistent view of the hot and dense medium. 


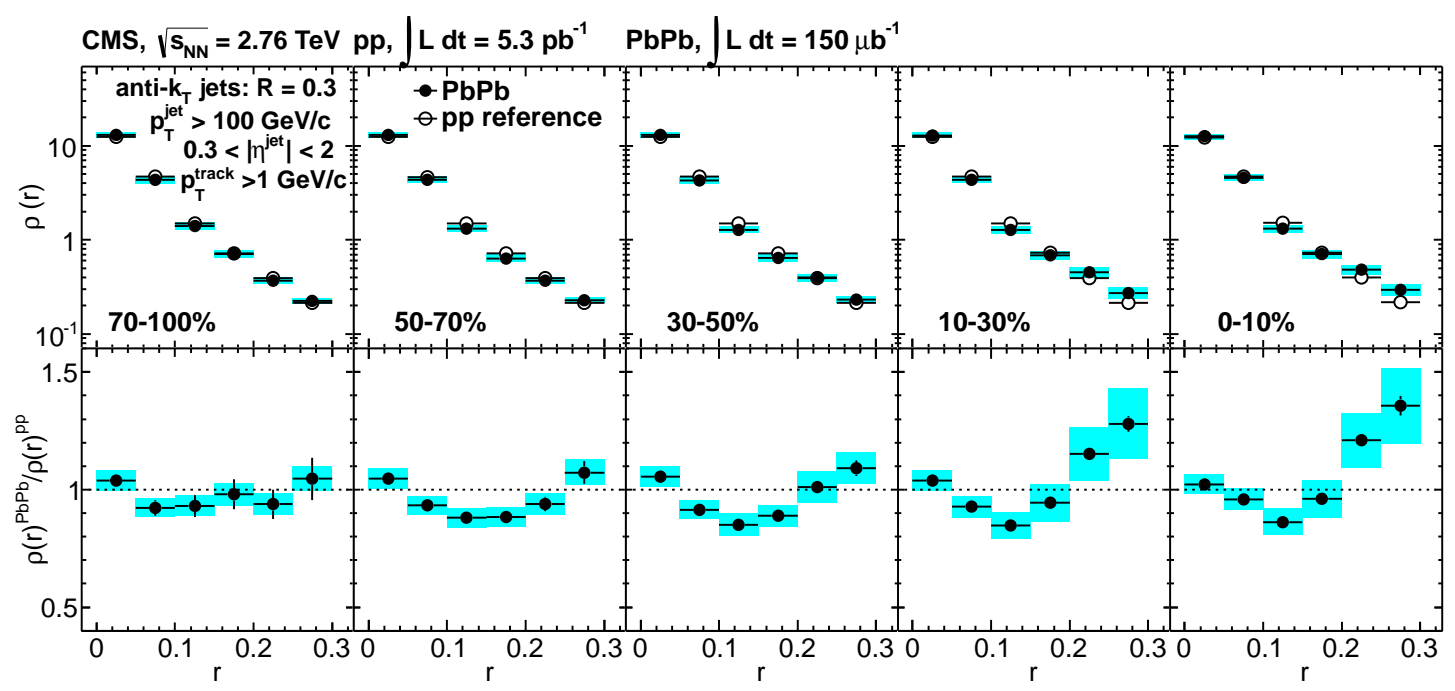

Figure 3: Differential jet shapes in $\mathrm{PbPb}$ and $\mathrm{pp}$ collisions are presented for different centrality bins for $p_{T}^{\text {jet }}>100 \mathrm{GeV} / \mathrm{c}$ with track $p_{T}>1 \mathrm{GeV}$ are shown in the top panels. Results from data are shown as black points while the open circles show the reference pp. In the bottom row, the ratio of the $\mathrm{PbPb}$ and $\mathrm{pp}$ jet shapes is shown. The blue band shows the total systematic uncertainty while the error bars indicate the statistical errors [9].

\section{References}

[1] J. D. Bjorken, "Energy loss of energetic partons in QGP: possible extinction of high $p_{T}$ jets in hadron-hadron collisions", (1982). FERMILAB-PUB-82-059-THY.

[2] CMS Collaboration, “The CMS experiment at the CERN LHC”, JINST 3 (2008) S08004.

[3] CMS Collaboration, "Observation and studies of jet quenching in $\mathrm{PbPb}$ collisions at $2.76 \mathrm{TeV}$ ", Phys. Rev. C 84 (2011) 024906.

[4] CMS Collaboration, "Jet momentum dependence of jet quenching in $\mathrm{PbPb}$ collisions at $\sqrt{s_{N N}}=2.76$ TeV’, Phys. Lett. B 712 (2012) 176.

[5] CMS Collaboration, "Studies of jet quenching using isolated-photon+jet correlations in $\mathrm{PbPb}$ and pp collisions at $\sqrt{s_{N N}}=2.76 \mathrm{TeV}$ ", Phys. Lett. B 18 (2013) 773.

[6] CMS Collaboration, "Measurement of the b-jet to inclusive jet ratio in $\mathrm{PbPb}$ and pp collisions at $\sqrt{s_{N N}}=2.76 \mathrm{TeV}$ with the CMS detector", CMS-PAS-HIN-12-003 (2012).

[7] CMS Collaboration, "Nuclear modification factor of high transverse momentum jets in $\mathrm{PbPb}$ collisions at $\sqrt{S_{N N}}=2.76 \mathrm{TeV}$ ', CMS-PAS-HIN-12-004 (2012).

[8] Vitev, I. et. al.,"A theory of jet shapes and cross sections”, JHEP 0811:093, 2008.

[9] CMS Collaboration, "Modification of jet shapes in PbPb collisions at $\sqrt{s_{N N}}=2.76 \mathrm{TeV}$ ", (2013) arXiv:1310.0878, submitted to PLB.

[10] CMS Collaboration, "Detailed Characterization of Jets in Heavy Ion Collisions Using Jet Shapes and JetFragmentation Functions”, (2012) CMS-PAS-HIN-12-013. 